\title{
DBI Phase Measurements of Amorphous Materials for Thickness and Structure Factor Determination
}

\author{
Rodney A. Herring ${ }^{1}$ \\ 1. University of Victoria, Canada
}

A method of diffracted beam interferometry (DBI) used to measure the mean inner potential of amorphous material epilayers is demonstrated with the potential to determine the amorphous material's structure factor [1]. An a-C epilayer on a single crystal Au substrate is used as an example. High contrast fringes were produced from the symmetric interference of the $220 \mathrm{~g}$ and $-2-2-2 \mathrm{~g}$ beams of $\mathrm{Au}$. The phase shifts within the interferogram due to the amorphous epilayers material are of interest because they represent the thickness and atomic structure factor, which to date has not been solved because of the missing phase information.

A phase measurement relative to the vacuum as the reference, the truly desired phase information necessary for determining the absolute thickness and structure factor of the amorphous material, is obtainable using this amplitude spitting method. In this method an amorphous epilayer exists or is placed on the surface of a thin crystal. Symmetric Bragg diffracted beams from the crystal are used to equally carry the amplitude and phase of the amorphous material. The two symmetric beams have equal but opposite phase, i.e., $\pm g$ hkl, which cancel when interfered using a biprism (Fig. 1) leaving two times the amorphous phase information,

$$
\Delta \phi=\phi_{g_{h k l}}+\phi_{\text {amor }}+\phi_{g_{\bar{h} \bar{k}}}+\phi_{\text {amor }}=2 \phi_{\text {amor }}
$$

A pseudo-planar beam passes through the amorphous layer, picks up its phase information, and is then carried through the thin crystal, which produces two carrier beams that are interfered as presented earlier. This method of beam interference offers the ability to accurately determine the thicknesses of the amorphous epilayer (Fig 2) and holds promise for determining the phase of short- and long-range structures of amorphous materials [3].

References:

[1] Herring R A et al, J. Electron Microscopy 61 (2012). p. 17.

[2] Herring, R.A., Microsc. Microanal. 21(3) (1957), p. 2015.

[3] Grants from UVic, NSERC, CFI and BCKDF are gratefully appreciated. 


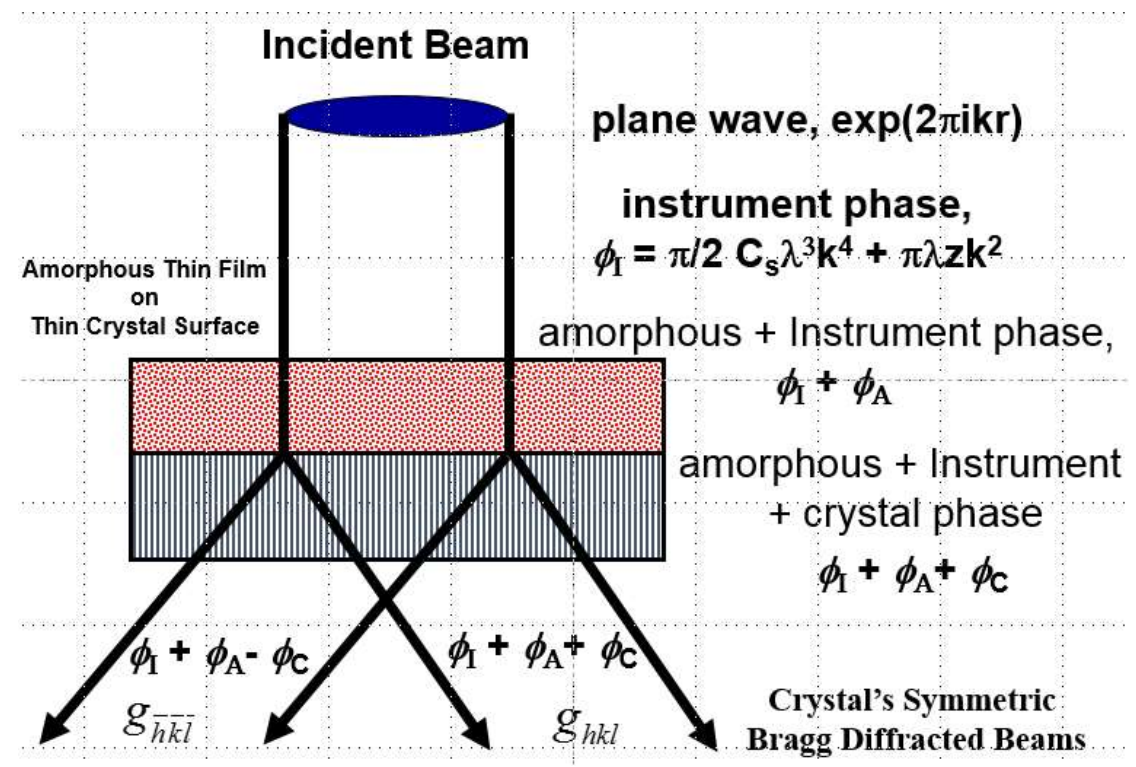

Figure 1. DBI of amorphous epilayer showing the amorphous phase information being carried by the crystal's Bragg diffracted beams resulting in the phase terms of equation 1.
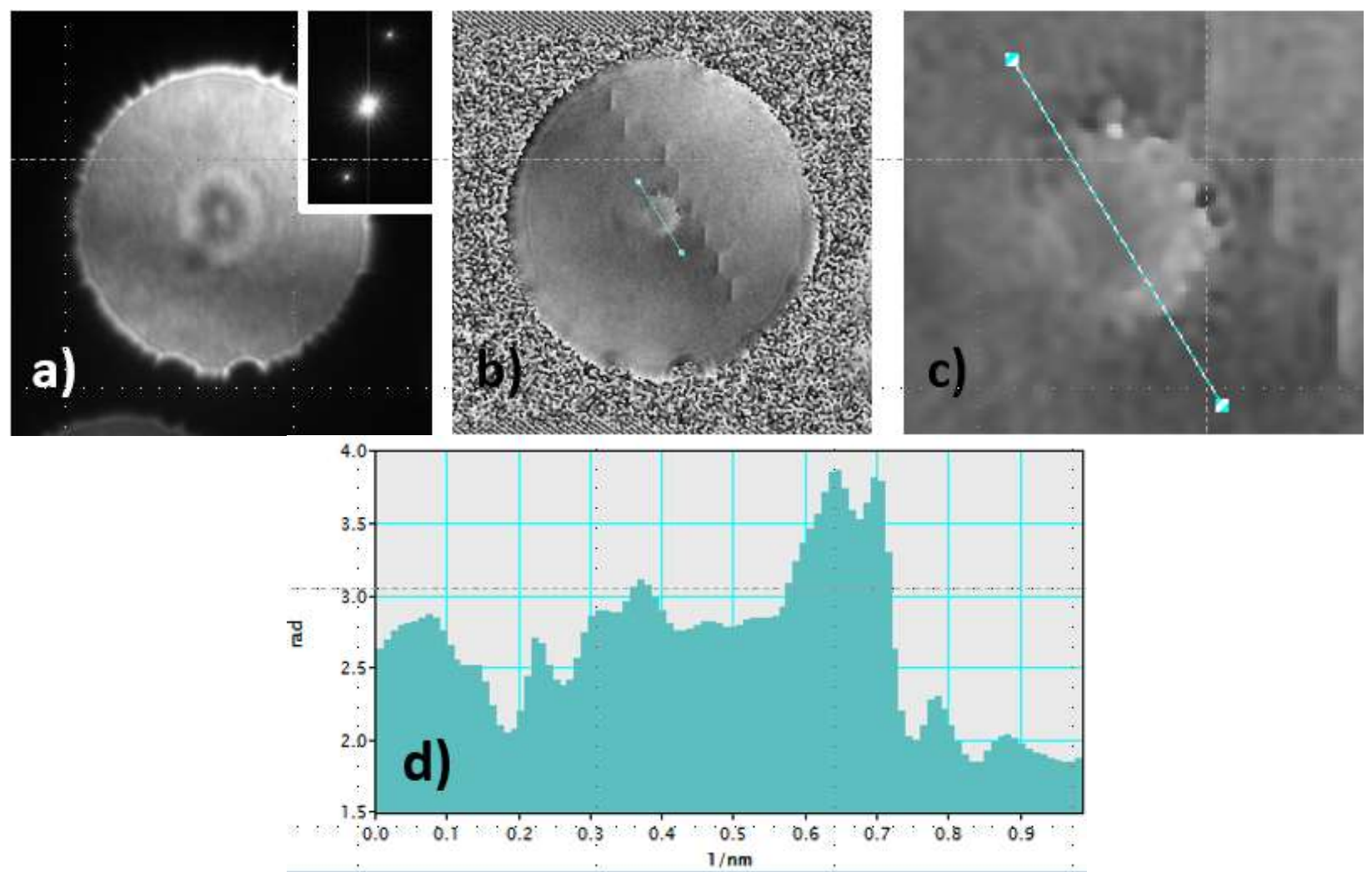

Figure 2. a) interferogram of two planar showing its Fourier transform in the inset, b) the retrieved phase information showing more clearly in c) the phase image of the a-C epilayers in the center (and the strain produced by a linear network of dislocations) producing in $\mathrm{d}$ ) a spatial measurement of the a-C phase information showing a thickness variation. 